\title{
Bidirectional associations between food groups and depressive symptoms: longitudinal findings from the Invecchiare in Chianti (InCHIANTI) study
}

\author{
Liset E. M. Elstgeest ${ }^{1 *}$, Marjolein Visser ${ }^{1}$, Brenda W. J. H. Penninx ${ }^{2}$, Marco Colpo ${ }^{3}$, Stefania Bandinelli ${ }^{3}$ \\ and Ingeborg A. Brouwer ${ }^{1}$ \\ ${ }^{1}$ Department of Health Sciences, Faculty of Science, Amsterdam Public Health research institute, Vrije Universiteit Amsterdam, \\ De Boelelaan 1085, 1081 HV Amsterdam, The Netherlands \\ ${ }^{2}$ Department of Psychiatry, Amsterdam Public Health research institute, Amsterdam UMC, Vrije Universiteit Amsterdam, \\ Oldenaller 1, $1081 \mathrm{HV}$ Amsterdam, The Netherlands \\ ${ }^{3}$ InCHIANTI Study Group, Azienda Sanitaria Toscana Centro, 50125 Florence, Italy
}

(Submitted 15 March 2018 - Final revision received 15 October 2018 - Accepted 16 October 2018)

\section{Abstract}

This study investigated bidirectional associations between intake of food groups and depressive symptoms in 1058 Italian participants (aged 20-102 years) of the Invecchiare in Chianti study. Dietary intake, assessed with a validated FFQ, and depressive symptoms, measured with the Center for Epidemiologic Studies Depression scale (CES-D), were assessed at baseline and after 3, 6 and 9 years. Associations of repeated measurements of intakes of thirteen food groups with 3-year changes in depressive symptoms, and vice versa, were analysed using linear mixed models and logistic generalised estimating equations. Fish intake was inversely (quartile (Q) $4 v$. Q1, $B=-0 \cdot 97,95 \% \mathrm{CI}-1 \cdot 74,-0 \cdot 21)$ and sweet food intake positively (Q4 $v$. Q1, $B=1 \cdot 03,95 \%$ CI $0 \cdot 25,1 \cdot 81)$ associated with subsequent CES-D score. In the other direction, higher CES-D scores were associated with decreases in intakes of vegetables (ratio: 0.995, 95\% CI 0.990, 0.999) and red and processed meat $(B=-0 \cdot 006,95 \% \mathrm{CI}-0 \cdot 010,-0 \cdot 001)$, an increase in dairy product intake (ratio: $1 \cdot 008,95 \%$ CI 1.004, 1.013), and increasing odds of eating savoury snacks (OR: 1.012, $95 \%$ CI 1.000, 1.024). Fruit, nuts and legumes, potatoes, wholegrain bread, olive oil, sugar-sweetened beverages, and coffee and tea were not significantly associated in either direction. Our study confirmed bidirectional associations between food group intakes and depressive symptoms. Fish and sweet food intakes were associated with 3-year improvement and deterioration in depressive symptoms, respectively. Depressive symptoms were associated with 3-year changes in vegetable, meat, dairy product and savoury snack intakes. Trials are necessary to examine the causal associations between food groups and depression.

Key words: Diet: Depression: Reverse causality: Older adults: Invecchiare in Chianti study

Nutritional epidemiology has traditionally focused on single nutrients and foods, but a complementary focus is on dietary patterns ${ }^{(1)}$. Dietary patterns as well as food groups have been linked to chronic diseases such as depression ${ }^{(2)}$. Reviews and meta-analyses on dietary patterns concluded that there seems to be a protective effect of 'healthy', 'traditional' and 'Mediterranean' dietary patterns, whereas a 'Western' dietary pattern may be associated with an increased risk of depression; however, they all emphasised that evidence for a true causal association is not yet conclusive, as findings were not consistent and residual confounding can still be present ${ }^{(3-7)}$. One of the reasons for the inconsistencies is the differences between studies on the used or derived dietary patterns and thus in the included components of these patterns. So by studying a priori and a posteriori patterns, it remains unknown which component(s) of the pattern are associated with health. It is therefore of interest to study these individual components, which are mostly food groups. Food groups can also be useful for communication in public health, and many dietary guidelines are food based ${ }^{(8,9)}$.

Reviews and meta-analyses regarding food groups as determinant ${ }^{(7,10,11)}$ - including fruit and vegetables ${ }^{(12,13)}$, fish $^{(14,15)}$ and coffee and tea ${ }^{(16)}$ - indicated some associations with depression as outcome, but their findings are inconsistent, potentially due to the limitations of the included individual studies. One of the limitations of many previous studies on diet and depression is their cross-sectional design. Although there are prospective studies on the link between food groups and depressive symptoms at follow-up measurements, they often only used dietary information at baseline, assuming no changes

Abbreviations: CES-D, Center for Epidemiologic Studies Depression scale; iADL, instrumental activities of daily living; InCHIANTI, Invecchiare in Chianti; LMM, linear mixed models; SSB, sugar-sweetened beverages and fruit juices.

* Corresponding author: L. E. M. Elstgeest, fax +31 205986 940, email liset.elstgeest@vu.nl 
in diet over time. To our knowledge, a few previous cohort studies used repeated measurements of dietary intake as determinant. Their repeated food group measurements were the average consumption at baseline and follow-up ${ }^{(17,18)}$, cumulative average of two time points ${ }^{(19)}$ or a categorical variable based on baseline consumption and 2-year change in consumption $^{(20)}$. Besides only three studies used longitudinal statistical methods to capture the repeated measurements of the exposure (i.e. food group) and found some prospective associations with incident depression outcomes for lower intakes at several measurements of fruit (but not vegetables) ${ }^{(21)}$, fruit and vegetables combined (unadjusted for other lifestyle factors) ${ }^{(22)}$, and higher intake of sugar in men only ${ }^{(23)}$.

The link between diet and depression may also exist in the other direction, that is, depressive symptoms may cause a change in the intake of food groups ('reverse causality'). Some studies have investigated the association of depression with food groups, dietary patterns and nutrients in this direction, but the majority of these studies were cross-sectional $^{(24-26)}$ or used retrospective depression data ${ }^{(27)}$. A total of three recent prospective studies also examined the reverse association and found depression to be associated with a lower fruit and vegetable intake (combined) (unadjusted for other lifestyle factors) ${ }^{(22)}$ but not with fish intake ${ }^{(17)}$ or sugar intake from sweet food/beverages ${ }^{(23)}$. However, to our knowledge, no other previous studies investigated the association of depression with food groups longitudinally.

Given the inconclusive and incomplete literature about the association between food groups and depression, and its direction, more prospective studies on the bidirectional link between diet and depression are needed. Except Smith et $a l .^{(17)}$, Kingsbury et $a l . .^{(22)}$ and Knüppel et al. ${ }^{(23)}$, no study has investigated this link in both directions within one cohort, which can give insight into the reverse causality hypothesis. These studies, as well as most studies on food groups, however, examined just one or two food groups, which does not allow comparison of the effect sizes of the associations with depression between several food groups.

In the InCHIANTI study (Invecchiare in Chianti, aging in the Chianti area), the link between dietary patterns and depressive symptoms has previously been studied. A Tuscan pattern at baseline derived by reduced rank regression (RRR) was cross sectionally and prospectively inversely associated with depressive symptoms ${ }^{(28)}$. However, two inflammatory dietary patterns at baseline - derived by RRR and characterised by unhealthy foods - were not prospectively associated with depressive symptoms ${ }^{(29)}$. We aim to extend this previous work by studying the individual components of these dietary patterns, that is, food groups. In addition, we use dietary data not only at baseline but also at follow-up measurements, and we address the relationship in two directions. The aims of this study were to study the prospective associations (1) between intake of food groups and change in depressive symptoms and (2) between depressive symptoms and changes in intake of food groups, in a large sample of Italian (older) adults.

\section{Methods}

\section{Study population}

The InCHIANTI study is a prospective, population-based cohort study of older adults in Tuscany (Italy) and was originally designed to examine factors that contribute to decline in mobility. In 1998-2000, a sample of 1453 persons was randomly recruited at two sites (Greve in Chianti and Bagno a Ripoli) using a multistage, stratified sampling method. A total of 1155 participants were aged 65-102 years, with those aged $\geq 90$ years oversampled, and 298 participants were aged 20-64 years. Baseline data were collected in a home interview and a medical evaluation at the study clinic. Follow-up measurements were performed 3, 6 and 9 years after baseline (respectively, 20012003, 2004-2006 and 2007-2009). A more detailed description of the study rationale, design and method is given elsewhere ${ }^{(30)}$. All participants signed informed consent after full explanation of procedures, and the ethics committee of the Italian National Institute of Research and Care on Aging approved the study protocol.

\section{Participants}

A total of 1206 persons had dietary data at baseline and at least at one of the three follow-up measurements. Persons with no or incomplete data on depressive symptoms at baseline or at any follow-up measurement were excluded ( $n$ 69). After exclusion of persons with missing data on baseline covariates (on physical activity ( $n$ 6) and waist circumference $(n 73)$ ), the analytic sample consisted of 1058 persons.

\section{Depression measurements}

At baseline and follow-up home visits, depressive symptoms were assessed with the Center for Epidemiologic Studies Depression scale (CES-D), a twenty-item self-report scale about depressive symptoms in the past week ${ }^{(31)}$. The CES-D, ranging from 0 to 60 points, is widely used and has been shown to be a valid instrument for assessing depressive symptoms in older adults $^{(32)}$ and in Italian adults ${ }^{(33)}$. The continuous CES-D scores were used as outcome or determinant in the associations, and the cut-off score of $\geq 20$ was used to indicate clinically relevant depressive symptoms for descriptive purposes. Although a cutoff of 16 is generally used, a higher cut-off has a greater specificity ${ }^{(32)}$ and seems to be more appropriate in Italian samples ${ }^{(33)}$.

\section{Dietary measurements}

At baseline and follow-up home visits, the usual dietary intake was assessed using the FFQ developed for the North-Central Italy centres ${ }^{(34)}$ of the European Prospective Investigation into Cancer and nutrition study ${ }^{(35)}$. This 240 -item FFQ on food consumption during the previous year has been validated for these centres ${ }^{(36)}$ as well as for the InCHIANTI cohort at population level ${ }^{(37)}$. Nutrient data were calculated using the Food Composition Database for Epidemiological Studies in Italy ${ }^{(38)}$. Based on the FFQ data obtained, thirteen food groups were a priori created: fruit, vegetables, nuts and legumes, potatoes, 
wholegrain bread, dairy products, red and processed meat, fish and shellfish, olive oil, savoury snacks, sweet foods, sugarsweetened beverages and fruit juices (SSB) and coffee and tea (online Supplementary Table S1). The selection of food groups was based on the fact that these are common components of diet-quality indices such as the Mediterranean Diet score, Alternative Healthy Eating Index and Dietary Approaches to Stop Hypertension Diet, or as previous studies on the depression-diet link suggested a link between the food group and depression (savoury snacks, sweet foods, coffee and tea) ${ }^{(16,19,23,25,39-41)}$. Intake in $\mathrm{g} / \mathrm{d}$ of each food group was calculated. Although the FFQ at the follow-up measurements differed somewhat from the FFQ at baseline, the food items included in the thirteen food groups were similar. The only considerable difference is that the items 'fruiting vegetables' and 'tomato sauce' in the baseline FFQ were redefined as 'tomatoes raw', 'tomatoes cooked' and 'other fruiting vegetables' in the follow-up FFQ.

\section{Other variables}

Data on potential confounders and variables used in sensitivity analyses were collected at the four measurements. Sociodemographic variables included age, sex, marital status (married, never married, widowed/divorced) and education level (years). Lifestyle factors included smoking status (never, former, current), alcohol intake ( $\mathrm{g} / \mathrm{d}$; from the FFQ) and total energy intake $(\mathrm{kJ} / \mathrm{d}$ and $\mathrm{kcal} / \mathrm{d}$, from the FFQ). In addition, physical activity level during the previous year was self-reported and classified as sedentary (hardly any physical activity or mostly sitting/some walking), light (light exercise $2-4 \mathrm{~h} /$ week) and moderate to intense (light physical activity $>4 \mathrm{~h} /$ week, moderate physical activity $>1 \mathrm{~h} /$ week or intense physical activity/ intense walks many times/week). Instrumental activities of daily living (iADL) disabilities were defined as self-report of inability or needing personal help in performing any basic or iADL; the number of disabilities was dichotomised $(0=$ no, $1-8=$ yes $)$. At the study clinic, waist circumference $(\mathrm{cm})$ was measured as well as body height and body weight that were used to calculate the BMI $\left(\mathrm{kg} / \mathrm{m}^{2}\right)$. Major diseases were ascertained according to standardised, pre-established criteria and algorithms based upon those used in the Women's Health and Aging Study ${ }^{(42)}$ using information on self-reported history, pharmacological treatments, medical examination data and hospital discharge records. These diseases included hypertension, CHD including angina pectoris and myocardial infarction, congestive heart failure, stroke, peripheral arterial disease, cancer, diabetes mellitus, chronic obstructive pulmonary disease, and hip and knee arthritis. The number of chronic diseases was categorised into no, 1 or 2 or more diseases. General cognitive functioning was assessed with the Mini Mental State Examination (MMSE), ranging from 0 to 30 points ${ }^{(43)}$. Use of antidepressants in the previous 2 weeks (yes/no) was coded according to the Anatomical Therapeutic Chemical classification system.

\section{Statistical analyses}

Baseline characteristics were described as means and standard deviations or medians and interquartile ranges and as percentages. Mean intakes of the food groups at baseline and at follow-up measurements were adjusted for energy intake, so that they are independent of total energy intake. This adjustment was performed using the residual method, where food group intakes are regressed on total energy intake ${ }^{(44)}$. Presented mean intakes are thus the predicted values from this regression. Spearman correlation coefficients were calculated between the food group intakes. For wholegrain bread, savoury snacks and SSB, the number of consumers was small. Therefore, the intakes of these food groups were dichotomised as non-consumers $v$. consumers.

The prospective associations of food group intakes as determinant with change in depressive symptoms as outcome were analysed by linear mixed models (LMM), which account for dependency of repeated measurements within individuals. The assumption of conditional normality of CES-D scores was assessed by analysis of residuals. A time-lag model was chosen by including intakes at baseline, follow-up 1 and follow-up 2, and CES-D scores at follow-up 1, follow-up 2 and follow-up 3 (both time-varying), resulting in three 3-year cycles. Baseline CES-D score was included as covariate to interpret the regression coefficients as change compared with baseline. Initially, the models included a random intercept, and random slopes for food group intake were added to the model and tested for improvement in fit. However, likelihood ratio tests did not show statistically significant improvements $(P<0.05)$ in fit for any food group, so the final models included only a random intercept. Standardised food group intake residuals were used as determinants to make comparisons between the food groups possible. Next to these continuous food group intake residuals, quartiles were made, with quartile 1 (lowest intake) serving as reference category. Quartiles of standardised intake residuals were defined for the four measurements, according to the distribution at baseline. To examine linear trends across the quartiles, the categorical quartile variable was used as a continuous variable to retrieve a $P$ value for trend. For the three dichotomous food groups, the non-consumer group was used as the reference category.

Effect modification by age and sex was tested by adding interaction terms between the standardised food group intake residuals and age or sex to the regression models. A $P$ value $<0 \cdot 10$ of an interaction term was considered statistically significant. Potential confounders were added to the univariable models and included in the final models if regression coefficients changed $>10 \%$ in at least one of the food groups. The following three models were made: model 1 was adjusted for baseline CES-D score, age, sex, marital status and education level; model 2 was additionally adjusted for physical activity, smoking, iADL disabilities, alcohol intake and energy intake; and model 3 was additionally adjusted for waist circumference and number of chronic diseases (as these variables might be confounders but more probably mediators). All confounders were included as time-varying variables (baseline, follow-up 1 and follow-up 2), except age, sex and education level (baseline). The analyses were repeated and additionally adjusted for the intake of the other food groups in a separate model to examine the independent association of the food groups with depressive symptoms. Last, time (years after baseline) and 
interaction terms between food group intake and time were added to the models to examine whether the associations varied with time.

The prospective associations of depressive symptoms as determinant with changes in continuous food group intake as outcome were analysed by LMM. Standardised food group intakes were used, which were checked for normality. As seven food groups were skewed to the right, a natural log transformation was performed on the intake at each measurement, after a value of 1 was added to prevent zero intakes. The regression coefficients $(B)$ and CI of the regression analyses were transformed back to obtain interpretable ratios $\left(e^{B}=\right.$ ratio). These ratios reflect the percentage of change in the outcome (standardised food group intake) per one unit change in the determinant (continuous CES-D score). A 3-year time-lag model was used by including CES-D scores at baseline, follow-up 1 and follow-up 2, and food group intakes at follow-up 1, follow-up 2 and follow-up 3. Baseline intake was included as covariate, and models with only random intercepts showed the best fit. Standardised intakes were used as outcomes to be able to compare between the food groups. For the three food groups used as dichotomous variables, logistic generalised estimating equations (GEE) with a logit link function and an exchangeable correlation structure were used. GEE take into account the correlation between repeated measurements in the same individual and is a more valid method than logistic mixed models for binary outcomes ${ }^{(45)}$. Effect modification, time interaction and confounding were handled as described above. The following three models were made: model 1 was adjusted for the baseline food group intake, age, sex, marital status and education level; model 2 was additionally adjusted for physical activity, smoking, iADL disabilities, alcohol intake and number of chronic diseases (as these variables might be confounders and/or mediators); and model 3 was additionally adjusted for energy intake and waist circumference (to examine the influence of these variables separately).

A total of three sensitivity analyses were performed in the LMM and GEE analyses by the exclusion of specific subgroups: (1) participants using antidepressants at any measurement, (2) participants with an MMSE score <24 at any measurement (who may have memory problems), and (3) participants with an implausible energy intake $(<2092 \mathrm{~kJ}(<500 \mathrm{kcal})$ or $>14644 \mathrm{~kJ}$ ( $>3500 \mathrm{kcal})$ for women and $<3347 \cdot 2 \mathrm{~kJ}$ ( $<800 \mathrm{kcal})$ or $\left(>16736 \mathrm{~kJ}(>4000 \mathrm{kcal})\right.$ for men) at any measurement ${ }^{(46)}$.

SPSS version 23 (SPSS Inc.) was used for all analyses. $P$ values $\leq 0.05$ were considered statistically significant.

\section{Results}

\section{Baseline characteristics}

Characteristics of the participants ( $54.7 \%$ women) are presented in Table 1. The mean age was 65.8 (sD 15.2) years, and 187 participants $(17 \cdot 7 \%)$ experienced clinically relevant depressive symptoms (CES-D $\geq 20$ ) at baseline. Comparison of included ( $n$ 1058) and excluded ( $n$ 395) participants showed that excluded participants were older $(P<0.001)$, less often married $(P<0.001)$, less educated $(P<0 \cdot 001)$, less physically active
Table 1. Baseline characteristics of the Invecchiare in Chianti study sample (1998-2000, n 1058)

(Mean values and standard deviations; numbers and percentages; medians and interquartile ranges (IQR))

\begin{tabular}{|c|c|c|c|}
\hline & $n$ & & $\%$ \\
\hline \multicolumn{4}{|l|}{ Age (years) } \\
\hline Mean & \multicolumn{3}{|c|}{$65 \cdot 8$} \\
\hline SD & \multicolumn{3}{|c|}{$15 \cdot 2$} \\
\hline Sex, women & 579 & & 55 \\
\hline \multicolumn{4}{|l|}{ Marital status } \\
\hline Married & 706 & & $66 \cdot 7$ \\
\hline Never married & 122 & & 11.5 \\
\hline Widowed/divorced & 230 & & $21 \cdot 7$ \\
\hline \multicolumn{4}{|l|}{ Education (years) } \\
\hline Mean & \multicolumn{3}{|c|}{6.9} \\
\hline SD & \multicolumn{3}{|c|}{$4 \cdot 2$} \\
\hline \multicolumn{4}{|l|}{ Physical activity } \\
\hline Sedentary & 127 & & $12 \cdot 0$ \\
\hline Light & 439 & & 41.5 \\
\hline Moderate to intense & 492 & & $46 \cdot 5$ \\
\hline \multicolumn{4}{|l|}{ Smoking } \\
\hline Never & 585 & & $55 \cdot 3$ \\
\hline Former & 262 & & 24.8 \\
\hline Current & 211 & & 19.9 \\
\hline iADL disabilities & 126 & & 12 \\
\hline \multicolumn{4}{|l|}{ Alcohol intake (g/d) } \\
\hline Median & \multicolumn{3}{|c|}{7.9} \\
\hline IQR & \multicolumn{3}{|c|}{$0 \cdot 2-26 \cdot 8$} \\
\hline \multicolumn{4}{|l|}{ BMI $\left(\mathrm{kg} / \mathrm{m}^{2}\right)$} \\
\hline Mean & \multicolumn{3}{|c|}{$27 \cdot 3$} \\
\hline SD & \multicolumn{3}{|c|}{$4 \cdot 1$} \\
\hline \multicolumn{4}{|l|}{ Waist circumference (cm) } \\
\hline Mean & \multicolumn{3}{|c|}{91.5} \\
\hline SD & \multicolumn{3}{|c|}{$11 \cdot 0$} \\
\hline \multicolumn{4}{|c|}{ Number of chronic diseases } \\
\hline 0 chronic disease & 365 & & 34.5 \\
\hline 1 chronic disease & 468 & & 44.2 \\
\hline$\geq 2$ chronic diseases & 225 & & $21 \cdot 3$ \\
\hline \multicolumn{4}{|c|}{ Cognitive functioning (MMSE score) } \\
\hline Mean & \multicolumn{3}{|c|}{$26 \cdot 4$} \\
\hline SD & \multicolumn{3}{|c|}{2.8} \\
\hline \multicolumn{4}{|c|}{ Depressive symptoms (CES-D score) } \\
\hline Mean & \multirow{2}{*}{\multicolumn{3}{|c|}{$\begin{array}{c}11.9 \\
8.5\end{array}$}} \\
\hline SD & & & \\
\hline Use of antidepressants & 44 & & $4 \cdot 2$ \\
\hline
\end{tabular}

iADL, instrumental activities of daily living; MMSE, Mini Mental State Examination; CES-D, Center for Epidemiologic Studies Depression scale.

$(P<0.001)$ and less often smokers $(P=0.021)$ compared with included participants. Excluded participants also had more iADL disabilities $(P<0.001)$, lower intakes of alcohol $(P<0.001)$ and energy $(P<0.001)$, lower cognitive function $(P<0.001)$ and more depressive symptoms $(P<0.001)$. Regarding food group intakes, excluded participants had lower intakes of fruit, vegetables, potatoes, red and processed meat, fish and shellfish and olive oil, and they ate less often wholegrain bread and savoury snacks (all $P<0.05$ ). These differences were most pronounced at baseline and follow-up 1 .

\section{Depressive symptoms and food group intakes over 9 years}

Depressive symptoms and energy and food group intakes at the four measurements are shown in Table 2. Clinically relevant depressive symptoms ranged from $17 \cdot 0$ to $24 \cdot 1 \%$. Total energy intake was highest after 3 years and lowest after 9 years. Energy-adjusted intakes of fruit, vegetables and olive oil 
Table 2. Center for Epidemiologic Studies Depression scale (CES-D) scores and intakes of energy and food groups of the Invecchiare in Chianti study sample ( $n$ 1058) at baseline and follow-up measurements

(Mean values and standard deviations; percentages)

\begin{tabular}{|c|c|c|c|c|c|c|c|c|}
\hline & \multicolumn{2}{|c|}{ Baseline } & \multicolumn{2}{|c|}{ Follow-up 1} & \multicolumn{2}{|c|}{ Follow-up 2} & \multicolumn{2}{|c|}{ Follow-up 3} \\
\hline & \multicolumn{2}{|c|}{$n 1058$} & \multicolumn{2}{|c|}{$n 960$} & \multicolumn{2}{|c|}{$n 853$} & \multicolumn{2}{|c|}{$n 757$} \\
\hline & Mean & SD & Mean & SD & Mean & SD & Mean & SD \\
\hline \multicolumn{9}{|l|}{ Depressive symptoms } \\
\hline Depressive symptoms (CES-D score) & 11.9 & 8.5 & $14 \cdot 3$ & $8 \cdot 6$ & $12 \cdot 9$ & $7 \cdot 6$ & $13 \cdot 5$ & $8 \cdot 1$ \\
\hline Presence of depressive symptoms (\%, CES-D $\geq 20$ ) & \multicolumn{2}{|c|}{$17 \cdot 7$} & \multicolumn{2}{|c|}{$24 \cdot 1$} & \multicolumn{2}{|c|}{$17 \cdot 0$} & \multicolumn{2}{|c|}{$19 \cdot 8$} \\
\hline \multicolumn{9}{|l|}{ Energy intake } \\
\hline $\mathrm{kJ} / \mathrm{d}$ & 8574 & 2598 & 8803 & 2595 & 8680 & 2638 & 8495 & 2579 \\
\hline $\mathrm{kcal} / \mathrm{d}$ & 2049 & 621 & 2104 & 620 & 2074 & 631 & 2030 & 617 \\
\hline \multicolumn{9}{|l|}{ Energy-adjusted intake - predicted values* $(\mathrm{g} / \mathrm{d})$} \\
\hline Fruit & 287 & 27 & 273 & 33 & 261 & 21 & 197 & 16 \\
\hline Vegetables & 184 & 38 & 139 & 34 & 129 & 35 & 124 & 31 \\
\hline Nuts and legumes & 18 & 1.6 & 17 & $1 \cdot 2$ & 16 & 2.4 & 20 & $2 \cdot 1$ \\
\hline Potatoes & 37 & 11 & 34 & $9 \cdot 7$ & 36 & 10 & 36 & $8 \cdot 4$ \\
\hline Dairy products & 174 & 29 & 178 & 23 & 171 & 13 & 191 & 18 \\
\hline Red and processed meat & 80 & 23 & 80 & 20 & 76 & 22 & 81 & 21 \\
\hline Fish and shellfish & 26 & 3.4 & 28 & $3 \cdot 8$ & 25 & 3.7 & 32 & 3.4 \\
\hline Olive oil & 27 & $6 \cdot 7$ & 26 & $6 \cdot 0$ & 23 & $6 \cdot 2$ & 23 & $5 \cdot 4$ \\
\hline Sweet foods & 69 & 25 & 72 & 27 & 65 & 18 & 86 & 41 \\
\hline Coffee and tea & 112 & 19 & 114 & 17 & 106 & $9 \cdot 4$ & 116 & $6 \cdot 7$ \\
\hline \multicolumn{9}{|l|}{ Consumers (\%) } \\
\hline Wholegrain bread & \multicolumn{2}{|c|}{$15 \cdot 2$} & \multicolumn{2}{|c|}{$16 \cdot 3$} & \multicolumn{2}{|c|}{$12 \cdot 7$} & \multicolumn{2}{|c|}{$25 \cdot 0$} \\
\hline Savoury snacks & \multicolumn{2}{|c|}{$31 \cdot 1$} & \multicolumn{2}{|c|}{28.5} & \multicolumn{2}{|c|}{$47 \cdot 2$} & \multicolumn{2}{|c|}{$32 \cdot 1$} \\
\hline Sugar-sweetened beverages + fruit juices & \multicolumn{2}{|c|}{$51 \cdot 8$} & \multicolumn{2}{|c|}{51.5} & \multicolumn{2}{|c|}{$55 \cdot 2$} & \multicolumn{2}{|c|}{$48 \cdot 7$} \\
\hline
\end{tabular}

* All predicted values are energy adjusted by regressing food group intake on total energy intake (the residual method).

decreased over time, while intakes of dairy products, fish and shellfish, and sweet foods increased, mainly between the 6- and 9 -year measurements. Intakes of nuts and legumes, potatoes, red and processed meat, and coffee and tea were quite stable over time. For wholegrain bread, the percentage of consumers ranged over time from $12 \cdot 7$ to $25 \cdot 0 \%$, and for savoury snacks, this was $28 \cdot 5-47 \cdot 2 \%$. SSB was consumed by about $50 \%$ of the participants across measurements. In online Supplementary Table S2, the correlations between the continuous and dichotomous food groups are shown.

\section{Food groups as determinants of change in depressive symptoms}

Results of the LMM analyses with food group intakes as determinants are shown in Table 3 and Fig. 1. No statistically significant associations were found after adjustment for confounders, except for two food groups. A positive association was found for the continuous standardised intake of sweet foods ( $B$, model 2: $0.30,95 \%$ CI $0.02,0.58$ ), showing that a higher intake of sweet foods was associated with an increase in depressive symptoms 3 years later (Fig. 1). The highest quartile of sweet foods was also positively associated compared with the lowest quartile, and a trend across quartiles was observed ( $P_{\text {for trend }}=0 \cdot 016$ ). For fish and shellfish, the highest quartile was associated with a subsequent decrease in depressive symptoms compared with the lowest quartile $(B=-0 \cdot 97,95 \%$ CI $-1.74,-0.21)$, and a trend was observed $\left(P_{\text {for trend }}=0.016\right)$ (Table 3). Unexpectedly, quartile 2 of potatoes was associated with less depressive symptoms compared with quartile 1 ( $B$, model 2: $-0 \cdot 75,95 \% \mathrm{CI}-1 \cdot 48,-0 \cdot 02$ ).
Additional adjustment for waist circumference and number of chronic diseases (model 3) did not change the findings. Significant interaction terms with sex were found for red and processed meat and with age for vegetables, nuts and legumes and SSB; however, after stratification by sex or the median of age, findings remained non-significant in all subgroups, and no clear trends were found (data not shown). For all continuous food groups, model 2 was additionally adjusted for the standardised intake residuals of the other nine continuous food groups; results did not change, except that the association of sweet foods attenuated $(B=0 \cdot 19,95 \% \mathrm{CI}-0 \cdot 11,0 \cdot 50)$. For the food group quartiles, additional adjustment did not change the findings, indicating that the associations are independent. Similarly, a model including simultaneously the three dichotomous food groups also showed similar results. For sweet foods, a significant, positive interaction with time $(P=0.018$ in model 2) was found, indicating that the association became stronger over time.

\section{Depressive symptoms as determinant of change in food groups}

Table 4 presents the results of the associations of depressive symptoms with change in food group intakes. The LMM analyses showed that a higher CES-D score was associated with 3 -year decreases in intakes of red and processed meat ( $B$ model 2: $-0.006,95 \% \mathrm{CI}-0 \cdot 010,-0 \cdot 001$ ) and vegetables (ratio: 0.995, $95 \%$ CI $0.990,0.999)$, and an increase in intake of dairy products (ratio: 1.008, 95\% CI 1.004, 1.013). The GEE analyses showed that a higher CES-D score was associated with a subsequent increase in odds of eating savoury snacks (OR: 1.012, 
Table 3. Prospective associations of intakes of food groups (determinants) in relation to change in Center for Epidemiologic Studies Depression scale (CES-D) score (outcome) 3 years later in the Invecchiare in Chianti study ( $n$ 1058, 9-year follow-up with 2467 observations) ${ }^{*}$

(Unstandardised regression coefficients and $95 \%$ confidence intervals)

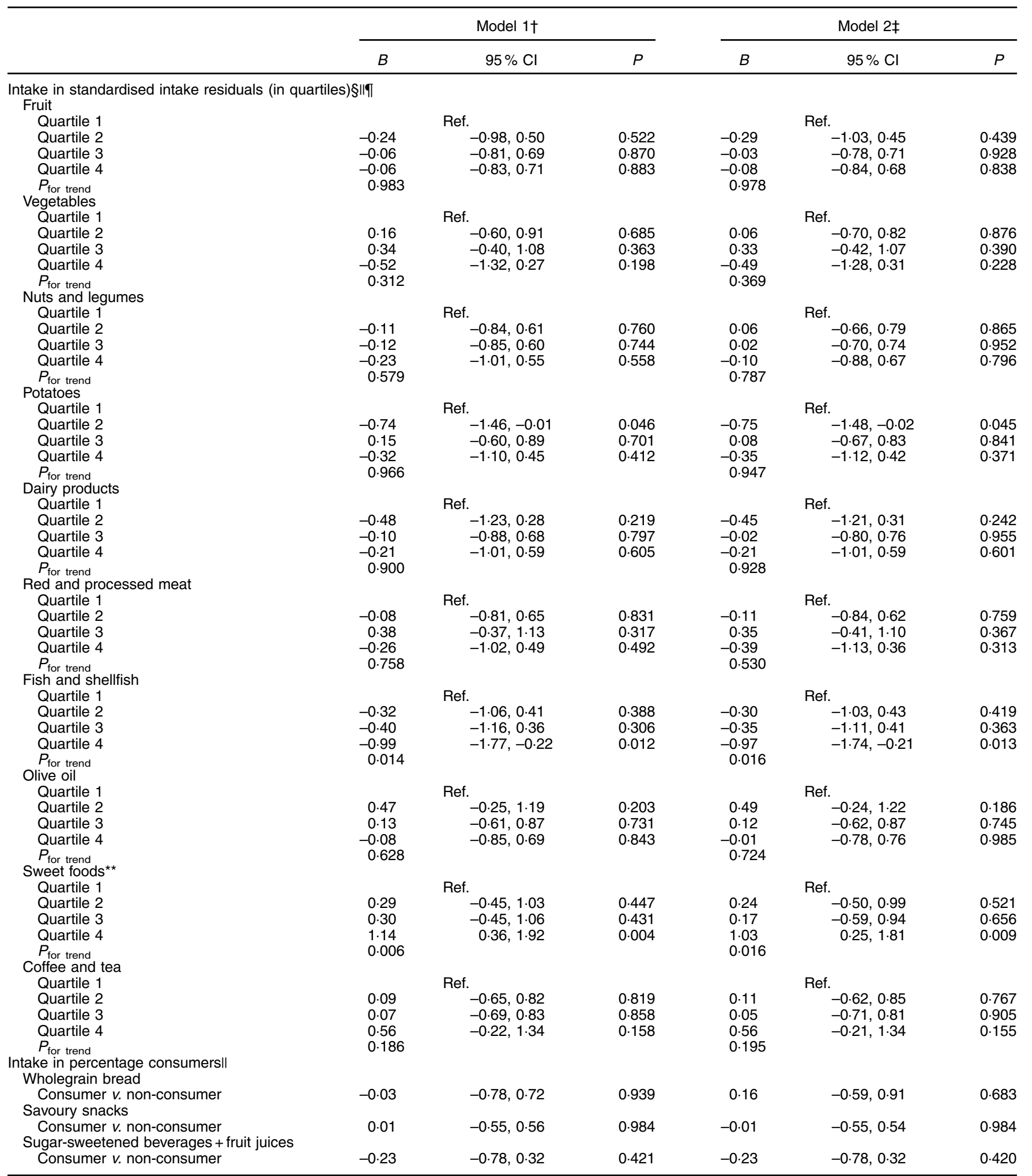

Ref., reference.

* Intake of food groups at baseline, follow-up 1 and follow-up 2, and CES-D scores at follow-up 1, 2 and 3.

† Model 1: adjusted for baseline CES-D score, age, sex, marital status and education level.

‡ Model 2: additionally adjusted for physical activity, smoking, instrumental activities of daily living disabilities, alcohol intake and energy intake.

$\S$ Intake residuals are energy adjusted by regressing food group intake on total energy intake (the residual method).

II Analysed with linear mixed models.

II Quartiles of the standardised intake residuals are based on the sample distribution at baseline.

${ }^{\star *}$ For sweet foods, there is a positive interaction with time, indicating that the association became stronger over time. 


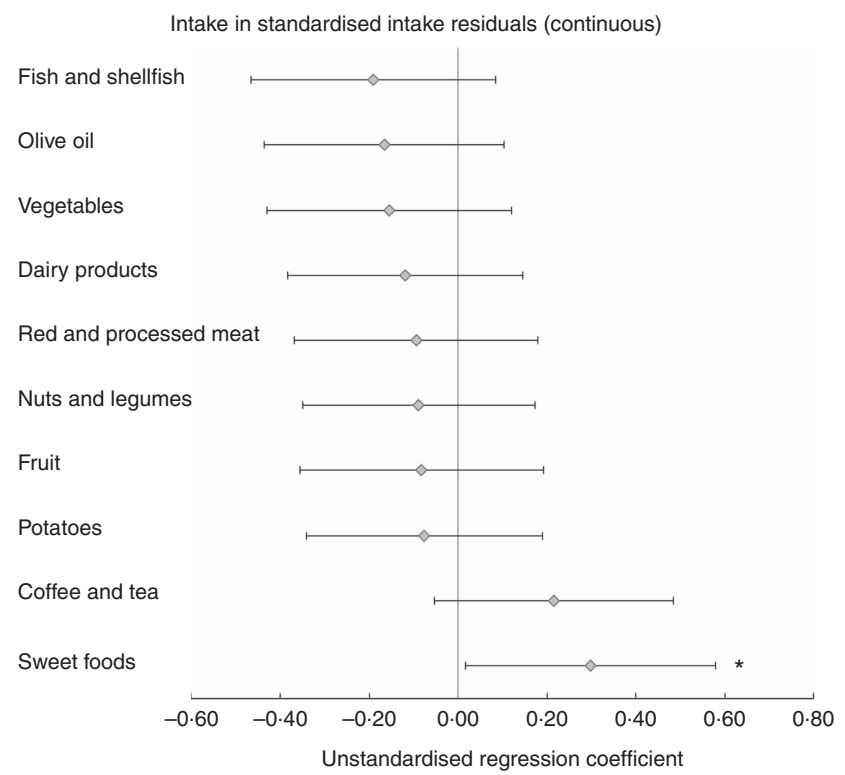

Fig. 1. Prospective associations of intake of food groups (determinants) in relation to change in Center for Epidemiologic Studies Depression scale (CES-D) score (outcome) 3 years later in the Invecchiare in Chianti study (n 1058, 9-year follow-up with 2467 observations). Intakes of food groups at baseline, follow-up 1 and follow-up 2, and CES-D scores at follow-up 1, 2 and 3. Intake residuals are energy adjusted by regressing food group intake on total energy intake (the residual method). Analysed with linear mixed models. Error bars indicate $95 \% \mathrm{Cl}$. Model 2 is shown: adjusted for baseline CES-D score, age, sex, marital status, education level, physical activity, smoking, iADL disabilities, alcohol intake and energy intake. For sweet foods, there is a positive interaction with time, indicating that the association became stronger over time. ${ }^{*} P<0.05$

$95 \%$ CI 1.000, 1.024). No statistically significant associations with the other food groups were found.

Additional adjustment for energy intake and waist circumference (model 3, observations $=2260$ ) did not change the findings; only the association with savoury snacks was slightly attenuated (OR: 1.011, 95\% CI 0.999, 1.024). Significant interaction terms with sex were found for coffee and tea and with age for vegetables, wholegrain bread, savoury snacks and SSB. After stratification by sex, still no significant associations with coffee and tea were found in men and women in model 2. Stratification by the median of age showed the same results for the mentioned food groups, except for vegetables: depressive symptoms were significantly inversely associated in older participants (those $>69$ years, ratio: $0.993,95 \%$ CI $0.987,1.000$ ) but not in younger participants $(0.996,95 \%$ CI 0.990, 1.002). Interactions with time were not significant in any of the food groups.

\section{Sensitivity analyses}

Findings of the food group intake-depressive symptoms analyses did not change when participants who had an implausible energy intake ( $n$ 36, observations $=93$ ) were excluded. However, the exclusion of participants who used antidepressants ( $n$ 226, observations $=673$ ) did not influence the fish association but resulted in a significant inverse association for the highest quartile of vegetables ( $B$ model $2:-1 \cdot 20,95 \% \mathrm{CI}-2 \cdot 12$, $-0 \cdot 28)$, and the association of sweet foods disappeared (continuous $B=0 \cdot 13,95 \% \mathrm{CI}-0 \cdot 19,0 \cdot 44$ ). Similarly, exclusion of participants who had an MMSE score $<24$ ( $n$ 667, observations $=1021$ ) did not influence the associations of fish and sweet foods but resulted in a significant inverse association for the highest vegetable quartile ( $B=-1.43,95 \% \mathrm{CI}-2 \cdot 44,-0 \cdot 43)$.

For the depressive symptoms-food group intake analyses, exclusion of participants who had an implausible energy intake ( $n$ 36, observations $=94$ ) did not change the findings. Further, exclusion of participants who used antidepressants ( $n$ 226, observations $=701$ ) attenuated the associations of red and processed meat ( $B$ model 2: $-0.004,95 \%$ CI -0.009 , 0.002), vegetables (ratio: $0.997,95 \%$ CI $0.992,1.003$ ) and savoury snacks (OR: 1.011, $95 \%$ CI 0.997, 1.025). Similarly, exclusion of participants who had an MMSE score <24 ( $n$ 667, observations $=1062$ ) led to statistically not significant associations of red and processed meat and vegetables.

\section{Discussion}

In this study, the bidirectional, prospective associations between thirteen food groups and depressive symptoms were investigated in 3-year intervals over a 9-year period. A high intake of fish and shellfish was prospectively associated with a decrease in depressive symptoms 3 years later, while a high intake of sweet foods was associated with an increase in depressive symptoms. In the other direction, more depressive symptoms were associated with subsequent decreases in the intakes of red and processed meat and vegetables, and increases in the intakes of dairy products and savoury snacks. For the other food groups, no associations were observed in either direction.

Associations were found in both directions; however, no food group had associations in both directions, which implies that reverse causality is not the reason for the observed associations in either direction. For example, the increase in depressive symptoms over 3 years in persons with a high sweet food intake is not due to the potential higher susceptibility to depression of these persons; this higher susceptibility could have led, already at baseline, to a higher sweet food intake. As we found no association of depressive symptoms with change in sweet food intake over time, there is no support for the reverse causality hypothesis. Besides we shaped the analyses in a 'prospective' way using a 3-year time-lag model and by adjustment for the outcome at baseline, thereby minimising the issue of 'cause or consequence'. So our prospective, bidirectional design made it possible to adequately investigate reverse causality; however, randomised controlled trials are needed to determine true causal relationships between food groups and depression. Prevention trials have not yet been performed, but two recent Australian treatment trials showed - in depressed adults - that dietary improvements had positive effects on mental health outcomes, including depression symptomatology. However, these trials had quite small samples (67 and 152 participants enroled) and did not primarily focus on food groups but on a Mediterranean-style diet, and its findings cannot be generalised to a more general population sample $e^{(47,48)}$

The associations of fish and sweet foods with depressive symptoms were strongest for the highest intake quartiles, while 
Table 4. Prospective associations of Center for Epidemiologic Studies Depression scale (CES-D) score (determinant) in relation to change in (logtransformed) intakes of food groups (outcomes) 3 years later in the Invecchiare in Chianti study ( $n$ 1058, 9-year follow-up with 2489 observations)* (Unstandardised regression coefficients, ratios, odds ratios and $95 \%$ confidence intervals)

\begin{tabular}{|c|c|c|c|c|c|c|}
\hline \multirow[b]{2}{*}{ Intake in standardised intake§ } & \multicolumn{3}{|c|}{ Model $1 \dagger$} & \multicolumn{3}{|c|}{ Model 2‡ } \\
\hline & $B$ & $95 \% \mathrm{Cl}$ & $P$ & $B$ & $95 \% \mathrm{Cl}$ & $P$ \\
\hline $\begin{array}{l}\text { Fruit } \\
\text { Red and processed meat } \\
\text { Olive oil }\end{array}$ & $\begin{array}{l}-0.004 \\
-0.006 \\
-0.004\end{array}$ & $\begin{array}{l}-0.009,0.001 \\
-0.011,-0.002 \\
-0.009,0.001\end{array}$ & $\begin{array}{l}0.140 \\
0.009 \\
0.081\end{array}$ & $\begin{array}{l}-0.003 \\
-0.006 \\
-0.004\end{array}$ & $\begin{array}{l}-0.008,0.002 \\
-0.010,-0.001 \\
-0.008,0.001\end{array}$ & $\begin{array}{l}0.313 \\
0.016 \\
0.136\end{array}$ \\
\hline $\begin{array}{l}\text { Intake in standardised, log- } \\
\text { transformed intake§ }\end{array}$ & Ratioll & $95 \% \mathrm{Cl}$ & $P$ & Ratioll & $95 \% \mathrm{Cl}$ & $P$ \\
\hline $\begin{array}{l}\text { Vegetables } \\
\text { Nuts and legumes } \\
\text { Potatoes } \\
\text { Dairy products } \\
\text { Fish and shellfish } \\
\text { Sweet foods } \\
\text { Coffee and tea }\end{array}$ & $\begin{array}{l}0.994 \\
0.995 \\
0.998 \\
1.007 \\
0.999 \\
1.003 \\
1.001\end{array}$ & $\begin{array}{l}0.989,0.998 \\
0.990,1.000 \\
0.993,1.003 \\
1.003,1.012 \\
0.994,1.004 \\
0.999,1.008 \\
0.996,1.005\end{array}$ & $\begin{array}{l}0.006 \\
0.051 \\
0.376 \\
0.001 \\
0.670 \\
0.120 \\
0.786\end{array}$ & $\begin{array}{l}0.995 \\
0.996 \\
0.998 \\
1.008 \\
0.999 \\
1.003 \\
1.001\end{array}$ & $\begin{array}{l}0.990,0.999 \\
0.991,1.001 \\
0.993,1.003 \\
1.004,1.013 \\
0.994,1.004 \\
0.998,1.007 \\
0.996,1.006\end{array}$ & $\begin{array}{r}0.022 \\
0.132 \\
0.375 \\
<0.001 \\
0.733 \\
0.233 \\
0.744\end{array}$ \\
\hline Intake in percentage consumers $\mathbb{f}$ & OR & $95 \% \mathrm{Cl}$ & $P$ & OR & $95 \% \mathrm{Cl}$ & $P$ \\
\hline $\begin{array}{l}\text { Wholegrain bread } \\
\text { Consumer } v \text {. non-consumer } \\
\text { Savoury snacks }\end{array}$ & 0.991 & $0.976,1.008$ & 0.294 & 0.993 & $0.976,1.010$ & 0.429 \\
\hline $\begin{array}{l}\text { Consumer } v \text {. non-consumer } \\
\text { Sugar-sweetened beverages + fruit juices }\end{array}$ & 1.012 & $1.001,1.023$ & 0.037 & 1.012 & $1.000,1.024$ & 0.047 \\
\hline Consumer v. non-consumer & 0.999 & $0.987,1.010$ & 0.815 & 0.996 & $0.983,1.008$ & 0.475 \\
\hline
\end{tabular}

* CES-D scores at baseline, follow-up 1 and follow-up 2, and intake of food groups at follow-up 1, 2 and 3.

† Model 1: adjusted for standardised (and log-transformed) baseline food group intake, age, sex, marital status and education level.

¥ Model 2: additionally adjusted for physical activity, smoking, instrumental activities of daily living disabilities, alcohol intake and number of chronic diseases.

$\S$ Analysed with linear mixed models.

II The ratios reflect the percentage of change in the outcome (standardised food group intake) per one unit change in the determinant (CES-D score).

If Analysed with generalised estimating equations.

the middle quartiles had comparable regression coefficients. This seems to indicate a non-linear association for both food groups, as shown before for fish ${ }^{(18,20)}$. Energy-adjusted intakes of fish and shellfish of more than $28.0 \mathrm{~g} / \mathrm{d}(\mathrm{Q} 4:>0.44$ standardised residuals $=>8.2$ residuals $=>28.0 \mathrm{~g} / \mathrm{d}$ predicted value $)$ and of sweet foods of more than $83.1 \mathrm{~g} / \mathrm{d}$ (Q4: $>0.43$ standardised residuals $=>20 \cdot 1$ residuals $=>83 \cdot 1 \mathrm{~g} / \mathrm{d}$ predicted value $)$ were associated with about one-point decrease and increase in CES-D score, respectively. All other food groups had regression coefficients of smaller sizes (non-significant), except for Q2 of potatoes. As there was no trend for the latter, this may represent a chance finding, potentially due to multiple testing. In the other direction, a one-point increase in CES-D score was associated with a reduced red and processed meat intake of $0.24 \mathrm{~g} / \mathrm{d}$ (regression coefficient: -0.006 standardised intake $=$ $-0.006 \times 41.2=0.24 \mathrm{~g} / \mathrm{d}$ ) and with a $0.8 \%$ increased dairy product intake (ratio: $1.008=$ increase of $0.8 \%$ ). So these effect sizes are relatively small. As we also did not adjust for multiple testing, our findings need to be interpreted with caution.

Another reason to be cautious is that our sensitivity analyses showed that some associations were not that robust. Exclusion of antidepressant users ( $n$ 226) made the association of sweet foods with depressive symptoms disappear. This might indicate that the positive sweet food association is mainly driven by those with more severe depression (who used antidepressants). In contrast, an inverse association of vegetables appeared, indicating that a high vegetable intake might lead to less depressive symptoms only in mentally healthy persons. Such beneficial association of vegetables has been shown in recent meta-analyses ${ }^{(7,12)}$. Nonetheless, the association of fish (quartile 4) remained significant. In the other direction, exclusion of antidepressant users ( $n$ 226) or participants with low-cognitive functioning ( $n$ 667) resulted in attenuation of the associations of depressive symptoms with red and processed meat, vegetables and savoury snacks. This might indicate that these associations mainly exist in persons with more severe depression or with low-cognitive functioning; however, a more plausible explanation for the attenuation is the smaller sample size that reduced the statistical power.

Our findings of the food group intake-depressive symptoms analyses are partly in line with previous studies. An inverse association between fish and depression risk has been described in recent meta-analyses of observational studies ${ }^{(7,14,15)}$ as well as in prospective studies in, for example, older Japanese ${ }^{(18)}$ and young Australian women ${ }^{(17)}$. A negative association between sweet foods and mood has also been found in prospective studies on commercial baked goods ${ }^{(41)}$, sugar intake from sweet food/beverages ${ }^{(23)}$ and added sugars ${ }^{(19)}$. Both findings are according to the hypothesis that 'healthy' foods lead to less depressive symptoms and 'unhealthy' foods to more depressive symptoms. However, we found no association for intakes of the other food groups that have previously been related to (incident) depression, including fruit ${ }^{(12,13,21,49-51)}$, vegetables $^{(7,12,13,49,51,52)}$, legumes ${ }^{(50)}$, meat ${ }^{(50)}$, grains ${ }^{(49)}$, dairy 
products $^{(50,53,54)}$ and coffee and tea ${ }^{(16,40)}$. It might be that for Italian older adults, other factors, such as sleep, socio-economic status, negative life events, social network or comorbidities $^{(55-57)}$, are more important risk factors for depression than these food groups, or that fish and sweet foods are key food groups that influence mood over time.

Two previous studies have been performed within InCHIANTI, using food intake data as determinant of depressive symptoms over time. Prospective associations have been shown between a Tuscan dietary pattern - characterised by vegetables, olive oil, fish, fruits, grains, potatoes, red and processed meat - and depressive symptoms ${ }^{(28)}$ but not between two inflammatory patterns and depressive symptoms ${ }^{(29)}$. These patterns were only measured at baseline, whereas we used intake data from baseline and after 3 and 6 years. Changes in food group intake over time thus seem to be important as we found no association between most Mediterranean food groups and depressive symptoms, except for fish. Similar to the unhealthy, inflammatory patterns, we found no association of unhealthy food groups with depressive symptoms, except for sweet foods. Vermeulen et $a l^{(29)}$ focused on patterns reflecting a mix of diverse food items - rather than single food groups, which may explain why they did not show this association. Also, they excluded participants aged $<65$ years, while we included these younger participants, resulting in a more varied dietary intake. So our study on food groups complements those of Vermeulen et al. ${ }^{(28,29)}$ on dietary patterns.

In the other direction, only a few previous prospective studies have been performed. We did not find an association between depressive symptoms and change in sweet food intake, similar to Knüppel et $a l .{ }^{(23)}$. We also did not find an association with fish intake as an outcome, which is in line with Smith et al. ${ }^{(17)}$, except that they showed a lower fish intake after 5 years in young women with a recent depression episode. The association of depression with fruit and vegetable intake (combined) was shown to be complex, since adjustment for lifestyle confounders (smoking, physical activity) attenuated the association in Canadian adults ${ }^{(22)}$; however, we investigated fruit and vegetables separately and found only an inverse association of depressive symptoms with vegetable intake, even after adjustment for smoking and physical activity. A comparison of our findings with other previous studies on the depression-diet link is rather difficult, since these studies were cross-sectional and did not investigate food groups comparable to ours ${ }^{(24-26)}$

Our finding that more depressive symptoms led to a higher consumption of savoury snacks is in line with the hypothesis that depressed persons may increase the consumption of less healthy (high-fat/salty) foods, owing to an increased appetite as in atypical depression ${ }^{(58)}$ or to a preference for consuming palatable 'comfort foods' ${ }^{\text {, }}$ '. In contrast, the association of depressive symptoms with a decreased intake of both red and processed meat and vegetables may reflect a reduced energy intake in depressed persons; this can result from a decreased appetite, which is one of the symptoms of the melancholic type of depression ${ }^{(60,61)}$ and common in older age $(75 \%$ of our study sample was aged $\geq 65$ years ${ }^{(62)}$. However, more depressive symptoms were also associated with increased intakes of dairy products and savoury snacks in our study, so this reduced energy intake in depressed persons must have been food group-specific. The increased intake of dairy products might be explained by a preference of depressed persons for easy-toprepare foods such as milk, yogurt and cheese ${ }^{(63)}$. Further, the null associations with intake of the other nine food groups might indicate that these food groups are not influenced by mood status.

Although the mechanistic understanding of the link between diet and depression is limited, several hypotheses exist. Diet influences physiologic processes, which consecutively influence development and progression of depression, such as neurotransmitter imbalances, neurogenesis and neuronal plasticity, oxidative stress and inflammation ${ }^{(57)}$. Regarding our findings, the protective effect of fish may be attributable not only to its $n$ - 3 fatty acids but also to other nutrients such as vitamin $\mathrm{D}$, vitamin $\mathrm{B}_{12}$, iodine and $\mathrm{Se}$; all play a role in the mentioned processes ${ }^{(64)}$. In the direction from depressive mood to diet, the underlying mechanisms might include sensory, physiological and psychological pathways ${ }^{(65)}$. Stress can influence food intake via physiological effects that change appetite or other eating behaviours, and it can also change food choices in persons with specific psychological characteristics such as emotional eaters ${ }^{(65)}$.

To our knowledge, this is the first study that investigated bidirectional associations between several food groups and depressive symptoms within one cohort. Most previous prospective studies investigated the unidirectional association from one food group to depression, and studies in the other direction with several dietary aspects as outcomes - were crosssectional $^{(24-26)}$. Another strength is the use of repeated measurements of outcome and determinant, that is, CES-D scores and food group intakes assessed four times over 9 years. Whereas previous diet-depression studies mostly used only baseline dietary data and assumed no changes over time, we took into account individual changes in food group intake over time using LMM. Furthermore, by using residual food group intakes as determinants and adjustment for energy intake, we were able to study the food group-depressive symptoms association independent of the energy intake. Finally, by studying food groups instead of dietary patterns or diet indices, we could identify the important components of these patterns/ indices that have a link with depressive symptoms.

Several limitations need to be addressed as well. In this Italian study population, intakes of three food groups (wholegrain bread, savoury snacks and SSB) were very low, compared with intakes in studies that showed association of these groups $^{(25,40,49)}$. Despite this small consumption range, we were able to pick up an association with savoury snacks as an outcome. Next, dietary data were derived by a self-reported FFQ: this assessment method, in combination with memory loss of the older participants, might lead to misreporting ${ }^{(66)}$; however, underreporting in older persons might partly reflect a true lowenergetic intake due to anorexia of ageing ${ }^{(67)}$. Depressive symptoms were also self-reported without a clinical assessment of depression, whereas a meta-analysis showed that healthy patterns and food groups were not associated with a formal diagnosis of depression, only with self-reported depressive 
symptoms $^{(7)}$. However, the CES-D scale is a validated instrument that has shown good psychometric properties in an older population ${ }^{(32,33)}$. Furthermore, residual confounding might be present attributable to imperfectly/not measured confounders such as the socio-economic status. Last, loss to follow-up is a limitation; the more healthy participants were available for this longitudinal study, which might have resulted in a less representative sample. This selection bias may have attenuated or strengthened our associations.

In summary, our study in an Italian (older) population, including repeated assessments of food group intakes and depressive symptoms, suggests that high intakes of fish and sweet foods are associated with, respectively, a decrease and increase in depressive symptoms. In the other direction, more depressive symptoms are associated with decreased intakes of red and processed meat and vegetables, and increased intakes of dairy products and savoury snacks. This bidirectional, prospective study confirms some of the hypothesised links between food groups and mood but not all. A bidirectional association between diet in general and depression seems to exist but not for specific food groups. Longitudinal studies on the association from depression to food group intakes are scarce, showing the need for more research. Future experimental studies should investigate the effects of a change in the intake of specific food groups on depression. This knowledge might be needed for the prevention of depression and the optimal nutritional care of depressed persons.

\section{Acknowledgements}

The authors would like to thank the participants of the InCHIANTI study for their continuing contributions and all participating departments of the InCHIANTI team. In addition, the authors would like to thank Prof. Jos Twisk for his advice regarding the statistical analyses.

Funding for this paper was provided by the European Union FP7 MooDFOOD Project 'Multi-country cOllaborative project on the rOle of Diet, FOod-related behaviour, and Obesity in the prevention of Depression' (grant agreement no. 613598).

The InCHIANTI study baseline (1998-2000) was supported as a 'targeted project' (ICS110.1/RF97.71) by the Italian Ministry of Health and in part by the US National Institute on Aging (contracts: 263 MD 916413 and 263 MD 821336); the InCHIANTI follow-up 1 (2001-2003) was funded by the US National Institute on Aging (contracts: N.1-AG-1-1 and N.1AG-1-2111); the InCHIANTI follow-ups 2 and 3 studies (20042010) were financed by the US National Institute on Aging (contract: N01-AG-5-0002); this research was supported in part by the Intramural Research Program of the National Institute on Aging, National Institutes of Health, Baltimore, Maryland.

The authors' responsibilities were as follows: L. E. M. E., I. A. B. and M. V. formulated the research questions and designed the study. L. E. M. E. conducted the statistical analyses, interpreted the data and drafted the manuscript. M. V., B. W. J. H. P., M. C., S. B. and I. A. B. contributed to the interpretation of the data and critically revised the manuscript. All authors approved the final manuscript.

The authors declare that there are no conflicts of interest.

\section{Supplementary material}

For supplementary material/s referred to in this article, please visit https://doi.org/10.1017/S0007114518003203

\section{References}

1. Hu FB (2002) Dietary pattern analysis: a new direction in nutritional epidemiology. Curr Opin Lipidol 13, 3-9.

2. Sánchez-Villegas A \& Martínez-González MA (2013) Diet, a new target to prevent depression? BMC Med 11, 3 .

3. Lai JS, Hiles S, Bisquera A, et al. (2014) A systematic review and meta-analysis of dietary patterns and depression in community-dwelling adults. Am J Clin Nutr 99, 181-197.

4. Psaltopoulou T, Sergentanis TN, Panagiotakos DB, et al. (2013) Mediterranean diet, stroke, cognitive impairment, and depression: a meta-analysis. Ann Neurol 74, 580-591.

5. Quirk SE, Williams LJ, O'Neil A, et al. (2013) The association between diet quality, dietary patterns and depression in adults: a systematic review. BMC Psychiatry 13, 175.

6. Rahe C, Unrath M \& Berger K (2014) Dietary patterns and the risk of depression in adults: a systematic review of observational studies. Eur J Clin Nutr 53, 997-1013.

7. Molendijk M, Molero P, Ortuno Sanchez-Pedreno F, et al. (2018) Diet quality and depression risk: a systematic review and dose-response meta-analysis of prospective studies. J Affect Disord 226, 346-354.

8. Montagnese C, Santarpia L, Buonifacio M, et al. (2015) European food-based dietary guidelines: a comparison and update. Nutrition 31, 908-915.

9. Gibney M \& Sandström B (2001) A framework for food-based dietary guidelines in the European Union. Public Health Nutr 4, 293-305.

10. Murakami K \& Sasaki S (2010) Dietary intake and depressive symptoms: a systematic review of observational studies. Mol Nutr Food Res 54, 471-488.

11. Sanhueza C, Ryan L \& Foxcroft DR (2013) Diet and the risk of unipolar depression in adults: systematic review of cohort studies. J Hum Nutr Diet 26, 56-70.

12. Liu X, Yan Y, Li F, et al. (2016) Fruit and vegetable consumption and the risk of depression: a meta-analysis. Nutrition 32, 296-302.

13. Saghafian F, Malmir H, Saneei P, et al. (2018) Fruit and vegetable consumption and risk of depression: accumulative evidence from an updated systematic review and metaanalysis of epidemiological studies. Br J Nutr 119, 1087-1101.

14. Li F, Liu X \& Zhang D (2016) Fish consumption and risk of depression: a meta-analysis. J Epidemiol Community Health 70, 299-304.

15. Grosso G, Micek A, Marventano S, et al. (2016) Dietary n-3 PUFA, fish consumption and depression: a systematic review and meta-analysis of observational studies. J Affect Dis $\mathbf{2 0 5}$, 269-281.

16. Grosso G, Micek A, Castellano S, et al. (2016) Coffee, tea, caffeine and risk of depression: a systematic review and doseresponse meta-analysis of observational studies. Mol Nutr Food Res 60, 223-234.

17. Smith KJ, Sanderson K, McNaughton SA, et al. (2014) Longitudinal associations between fish consumption and depression in young adults. Am J Epidemiol 179, 1228-1235.

18. Matsuoka YJ, Sawada N, Mimura M, et al. (2017) Dietary fish, n-3 polyunsaturated fatty acid consumption, and depression risk in Japan: a population-based prospective cohort study. Transl Psychiatry 7, e1242.

19. Sánchez-Villegas A, Zazpe I, Santiago S, et al. (2018) Added sugars and sugar-sweetened beverage consumption, dietary 
carbohydrate index and depression risk in the Seguimiento Universidad de Navarra (SUN) Project. Br J Nutr 119, 211-221.

20. Sánchez-Villegas A, Henríquez P, Figueiras A, et al. (2007) Long chain omega-3 fatty acids intake, fish consumption and mental disorders in the SUN cohort study. Eur J Clin Nutr $\mathbf{4 6}$, 337-346.

21. Mihrshahi S, Dobson AJ \& Mishra GD (2015) Fruit and vegetable consumption and prevalence and incidence of depressive symptoms in mid-age women: results from the Australian longitudinal study on women's health. Eur J Clin Nutr 69, 585-591.

22. Kingsbury M, Dupuis G, Jacka F, et al. (2016) Associations between fruit and vegetable consumption and depressive symptoms: evidence from a national Canadian longitudinal survey. J Epidemiol Community Health 70, 155-161.

23. Knüppel A, Shipley MJ, Llewellyn CH, et al. (2017) Sugar intake from sweet food and beverages, common mental disorder and depression: prospective findings from the Whitehall II study. Sci Rep 7, 6287.

24. Appelhans BM, Whited MC, Schneider KL, et al. (2012) Depression severity, diet quality, and physical activity in women with obesity and depression. J Acad Nutr Diet 112, 693-698.

25. Jeffery RW, Linde JA, Simon GE, et al. (2009) Reported food choices in older women in relation to body mass index and depressive symptoms. Appetite 52, 238-240.

26. Whitaker KM, Sharpe PA, Wilcox S, et al. (2014) Depressive symptoms are associated with dietary intake but not physical activity among overweight and obese women from disadvantaged neighborhoods. Nutr Res 34, 294-301.

27. Jacka FN, Cherbuin N, Anstey KJ, et al. (2015) Does reverse causality explain the relationship between diet and depression? J Affect Dis 175, 248-250.

28. Vermeulen E, Stronks K, Visser M, et al. (2016) The association between dietary patterns derived by reduced rank regression and depressive symptoms over time: the Invecchiare in Chianti (InCHIANTI) study. Br J Nutr 115, 2145-2153.

29. Vermeulen E, Brouwer IA, Stronks K, et al. (2018) Inflammatory dietary patterns and depressive symptoms in Italian older adults. Brain Behav Immun 67, 290-298.

30. Ferrucci L, Bandinelli S, Benvenuti E, et al. (2000) Subsystems contributing to the decline in ability to walk: bridging the gap between epidemiology and geriatric practice in the InCHIANTI study. J Am Geriatr Soc 48, 1618-1625.

31. Radloff LS (1977) The CES-D scale: a self-report depression scale for research in the general population. Appl Psychol Meas 1, 385-401.

32. Beekman ATF, Deeg DJH, Van Limbeek J, et al. (1997) Criterion validity of the Center for Epidemiologic Studies Depression scale (CES-D): results from a community-based sample of older subjects in the Netherlands. Psychol Med 27, 231-235.

33. Fava GA (1983) Assessing depressive symptoms across cultures: Italian validation of the CES-D self-rating scale. $J$ Clin Psychol 39, 249-251.

34. Pala V, Sieri S, Palli D, et al. (2003) Diet in the Italian EPIC cohorts: presentation of data and methodological issues. Tumori 89, 594-607.

35. Riboli E, Hunt KJ, Slimani N, et al. (2002) European Prospective Investigation into Cancer and Nutrition (EPIC): study populations and data collection. Public Health Nutr 5, 1113-1124.

36. Pisani P, Faggiano F, Krogh V, et al. (1997) Relative validity and reproducibility of a food frequency dietary questionnaire for use in the Italian EPIC centres. Int I Epidemiol $\mathbf{2 6}$ S152-S160.

37. Bartali B, Turrini A, Salvini S, et al. (2004) Dietary intake estimated using different methods in two Italian older populations. Arch Gerontol Geriatr 38, 51-60.

38. Salvini S, Parpinel M, Gnagnarella P, et al. (1998) Banca dati di composizione degli alimenti per studi epidemiologici in Italia (Food Composition Database for Epidemiological Studies in Italy). Milano: Istituto Europeo di Oncologia.

39. Konttinen H, Männistö S, Sarlio-Lähteenkorva S, et al. (2010) Emotional eating, depressive symptoms and self-reported food consumption. A population-based study. Appetite 54, 473-479.

40. Guo X, Park Y, Freedman ND, et al. (2014) Sweetened beverages, coffee, and tea and depression risk among older US adults. PLOS ONE 9, e94715.

41. Sánchez-Villegas A, Toledo E, de Irala J, et al. (2012) Fast-food and commercial baked goods consumption and the risk of depression. Public Health Nutr 15, 424-432.

42. Guralnik JM, Fried LP, Simonsick EM, et al. (1995) The Women's Health and Aging Study: Health and Social Characteristics of Older Women with Disability. NIH Publication No. 95-4009 ed., NIH Publication No 95-4009. Bethesda, MD: National Institute on Aging.

43. Folstein MF, Folstein SE \& McHugh PR (1975) "Mini-mental state". A practical method for grading the cognitive state of patients for the clinician. J Psychiatr Res 12, 189-198.

44. Willett WC, Howe GR \& Kushi LH (1997) Adjustment for total energy intake in epidemiologic studies. Am J Clin Nutr $\mathbf{6 5}$, 1220S-1228S.

45. Twisk JWR, de Vente W, Apeldoorn AT, et al. (2017) Should we use logistic mixed model analysis for the effect estimation in a longitudinal RCT with a dichotomous outcome variable? Epidemiol Biostat Public Health 14, e12613.

46. Willett WC (1998) Issues in analysis and presentation of dietary data. In Nutritional Epidemiology, 2nd ed., pp. 321-346 [WC Willett, editor]. New York, NY: Oxford University Press.

47. Jacka FN, O'Neil A, Opie R, et al. (2017) A randomised controlled trial of dietary improvement for adults with major depression (the 'SMILES' trial). BMC Med 15, 23.

48. Parletta N, Zarnowiecki D, Cho J, et al. (2017) A Mediterraneanstyle dietary intervention supplemented with fish oil improves diet quality and mental health in people with depression: a randomized controlled trial (HELFIMED). Nutr Neurosci (epublication ahead of print version 7 December 2017).

49. Gangwisch JE, Hale L, Garcia L, et al. (2015) High glycemic index diet as a risk factor for depression: analyses from the Women's Health Initiative. Am J Clin Nutr 102, 454-463.

50. Sánchez-Villegas A, Delgado-Rodriguez M, Alonso A, et al. (2009) Association of the Mediterranean dietary pattern with the incidence of depression: the Seguimiento Universidad de Navarra/University of Navarra follow-up (SUN) cohort. Arch Gen Psychiatry 66, 1090-1098.

51. Akbaraly TN, Sabia S, Shipley MJ, et al. (2013) Adherence to healthy dietary guidelines and future depressive symptoms: evidence for sex differentials in the Whitehall II study. Am J Clin Nutr 97, 419-427.

52. Tsai AC, Chang T-L \& Chi S-H (2012) Frequent consumption of vegetables predicts lower risk of depression in older Taiwanese - results of a prospective population-based study. Public Health Nutr 15, 1087-1092.

53. Pasco JA, Williams LJ, Brennan-Olsen SL, et al. (2015) Milk consumption and the risk for incident major depressive disorder. Psychother Psychosom 84, 384-386.

54. Perez-Cornago A, Sanchez-Villegas A, Bes-Rastrollo M, et al. (2016) Intake of high-fat yogurt, but not of low-fat yogurt or 
prebiotics, is related to lower risk of depression in women of the SUN cohort study. J Nutr 146, 1731-1739.

55. Djernes JK (2006) Prevalence and predictors of depression in populations of elderly: a review. Acta Psychiatr Scand 113, 372-387.

56. Vink D, Aartsen MJ \& Schoevers RA (2008) Risk factors for anxiety and depression in the elderly: a review. J Affect Dis 106, 29-44.

57. Lopresti AL, Hood SD \& Drummond PD (2013) A review of lifestyle factors that contribute to important pathways associated with major depression: diet, sleep and exercise. $J$ Affect Dis 148, 12-27.

58. Rahe C, Baune BT, Unrath M, et al. (2015) Associations between depression subtypes, depression severity and diet quality: cross-sectional findings from the BiDirect Study. BMC Psychiatry 15, 38.

59. Singh M (2014) Mood, food, and obesity. Front Psychol 5, 925.

60. American Psychiatric Association (2013) Diagnostic and Statistical Manual of Mental Disorders, 5th ed. Washington, DC.

61. Penninx B, Milaneschi Y, Lamers F, et al. (2013) Understanding the somatic consequences of depression: biological mechanisms and the role of depression symptom profile. $B M C$ Med 11, 129.

62. Donini LM, Savina C \& Cannella C (2003) Eating habits and appetite control in the elderly: the anorexia of aging. Int Psychogeriatr 15, 73-87.

63. Davison KM \& Kaplan BJ (2012) Food intake and blood cholesterol levels of community-based adults with mood disorders. BMC Psychiatry 12, 10.

64. Grosso G, Galvano F, Marventano S, et al. (2014) Omega-3 fatty acids and depression: scientific evidence and biological mechanisms. Oxid Med Cell Longev 2014, 313570.

65. Gibson EL (2006) Emotional influences on food choice: sensory, physiological and psychological pathways. Physiol Behav 89, 53-61.

66. McNeill G, Winter J \& Jia X (2009) Diet and cognitive function in later life: a challenge for nutrition epidemiology. Eur J Clin Nutr 63, S33-S37.

67. Shahar DR, Yu B, Houston DK, et al. (2010) Misreporting of energy intake in the elderly using doubly labeled water to measure total energy expenditure and weight change. $J \mathrm{Am}$ Coll Nutr 29, 14-24.

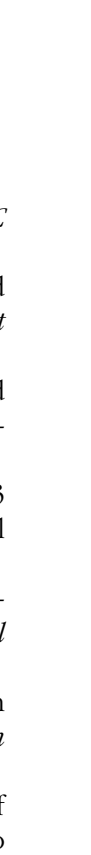

